\title{
Improved Langevin Approach to Spinodal Decomposition in the Chiral Transition
}

\author{
Eduardo S. Fraga*, Gastão Krein ${ }^{\dagger}$ and Rudnei O. Ramos** \\ * Instituto de Física, Universidade Federal do Rio de Janeiro \\ C.P. 68528, 21941-972 Rio de Janeiro, RJ , Brazil \\ †Instituto de Física Teórica, Universidade Estadual Paulista \\ Rua Pamplona 145, 01405-900 São Paulo, SP, Brazil \\ ** Departamento de Física Teórica, Universidade do Estado do Rio de Janeiro, \\ 20550-013 Rio de Janeiro, RJ Brazil
}

\begin{abstract}
We use an improved Langevin description that incorporates both additive and multiplicative noise terms to study the dynamics of phase ordering. We perform real-time lattice simulations to investigate the role played by different contributions to the dissipation and noise. Lattice-size independence is assured by the use of appropriate lattice counterterms.
\end{abstract}

Keywords: QCD phase transition; Heavy-ion collisions; Dissipation in field theory PACS: 25.75.-q, 11.30.Rd, 05.70.-a, 64.90.+b

The dynamics of spinodal decomposition after a phase transition in field theory plays a major role in physics, for instance in the nonequilibrium time evolution of order parameters [1]. The influence of the presence of a hot and dense medium on the dynamics of particles and fields is encoded "macroscopically" in attributes such as dissipation and noise, and can dramatically affect the relevant time scales for different stages of phase conversion. In particular, in high-energy heavy ion collisions, where one presumably forms a hot, dense, strongly interacting quark-gluon plasma [2], chiral fields evolve under extreme conditions of temperature and density during the QCD phase transition. To have a clear understanding of data coming from BNL-RHIC, and especially of data that will be produced at CERN-LHC, one needs a realistic description of the hierarchy of scales associated with dissipation, noise, radiation, expansion and finite size of the system.

Recently, some of us have considered the effects of dissipation in the scenario of explosive spinodal decomposition for hadron production $[3,4,5,6,7]$ during the QCD transition after a high-energy heavy ion collision in the simplest fashion [8]. Using a phenomenological Langevin description for the time evolution of the order parameter in a chiral effective model [9], inspired by microscopic nonequilibrium field theory results $[10,11,12]$, we performed real-time lattice simulations for the behavior of the inhomogeneous chiral fields. It was shown that the effects of dissipation could be dramatic in spite of very conservative assumptions: even if the system quickly reaches the unstable region there is still no guarantee that it will explode. The framework for the dynamics was assumed to be given by the following Langevin equation:

$$
\frac{\partial^{2} \phi}{\partial t^{2}}-\nabla^{2} \phi+\eta \frac{\partial \phi}{\partial t}+\frac{d V_{\mathrm{eff}}(\phi)}{d \phi}=\xi(\vec{x}, t),
$$


where $\phi$ is a real scalar field, $V_{\text {eff }}(\phi)$ is a Landau-Ginzburg effective potential and $\eta$, which can be seen as a response coefficient that defines time scales for the system and encodes the intensity of dissipation, is usually taken to be a function of temperature only, $\eta=\eta(T)$. The function $\xi(\vec{x}, t)$ represents a stochastic (noise) force, assumed Gaussian and white, so that $\langle\xi(\vec{x}, t)\rangle=0$ and $\left\langle\xi(\vec{x}, t) \xi\left(\overrightarrow{x^{\prime}}, t^{\prime}\right)\right\rangle=2 \eta T \delta\left(\vec{x}-\overrightarrow{x^{\prime}}\right) \delta\left(t-t^{\prime}\right)$, according to the fluctuation-dissipation theorem.

Although phenomenological equations of the form of Eq. (1) are widely used in the literature, formal derivations of effective equations of motion based on a more complete field-theoretic description of nonequilibrium dissipative dynamics [10, 11, 12, 13] show that much more complicated equations emerge. In general they are nonlocal equations of motion, with colored, non-Markovian dissipative and noise kernels, exhibiting both noise and dissipation terms that depend on the field amplitude. It is then important to analyze the possible outcome of dynamics emerging from these more realistic equations and compare them to the simple phenomenological equation (1). In fact, on very general physical grounds, one expects that dissipation effects should depend on the local density $\sim \phi^{2} \dot{\phi}$ and, accordingly, the noise term should contain a multiplicative piece $\sim \phi$. These results emerge as appropriate approximations to the effective equations of motion derived e.g. in Refs. [10, 11, 13].

Motivated by previous results for effective equations of motion obtained from microscopic derivations $[10,11,13]$, we consider an improved Langevin framework which includes the effects of multiplicative noise and density-dependent dissipation terms in the description of the time evolution of the order parameter, which should be closer to a more realistic physical realization than the one described by Eq. (1). To assess the effect of the new contributions on the relevant time scales for phase ordering, we consider a scalar $\lambda \phi^{4}$ theory in the broken phase. It was shown recently that the effects of multiplicative noise are rather significant in the Kibble-Zurek scenario of defect formation in one spatial dimension [14]. We perform $(3+1)$-dimensional real-time lattice simulations to study the behavior of the inhomogeneous scalar field, taking into account lattice counter-terms that guarantee lattice-size independence $[15,16,17]$.

To model the mechanism of phase ordering we adopt a $\lambda \phi^{4}$ theory in the broken phase, given by the standard Lagrangian density

$$
\mathscr{L}=\frac{1}{2}\left(\partial_{\mu} \phi\right)\left(\partial^{\mu} \phi\right)+\frac{1}{2} m^{2} \phi^{2}-\lambda \phi^{4},
$$

where $\phi$ is a scalar field playing the role of the order parameter and $\lambda>0$. In what follows we assume that the system was quenched from a high-temperature environment in which the sign of the mass term was the opposite, corresponding to a symmetric phase, down to a temperature below some critical temperature of symmetry restoration $\left(T<T_{c}\right)$, in which the effective potential has the form shown in the Lagrangian density above.

In our analysis, the time evolution of the field $\phi(\vec{x}, t)$ at each point in space and its approach to equilibrium will be dictated by an improved Langevin equation of the form

$$
\left(\frac{\partial^{2}}{\partial t^{2}}-\nabla^{2}\right) \phi(\vec{x}, t)+\left[\eta_{1}+\eta_{2} \phi^{2}(\vec{x}, t)\right] \frac{\partial \phi(\vec{x}, t)}{\partial t}+V^{\prime}(\phi)=\xi_{1}(\vec{x}, t)+\phi(\vec{x}, t) \xi_{2}(\vec{x}, t),
$$


which models the local approximation for the effective equation of motion derived e.g. in Ref. [10]. In Eq. (3), $\eta_{1}$ and $\eta_{2}$ will be taken to be functions of temperature only, $\eta_{i}=\eta_{i}(T)$. The functions $\xi_{1}(\vec{x}, t)$ and $\xi_{2}(\vec{x}, t)$ represent stochastic (noise) forces, assumed Gaussian and white.

Eq. (3) could, in principle, be obtained from a microscopic field-theoretic description of the real-time nonequilibrium dynamics of the chiral field at finite temperature [10, 12]. The noise and dissipation terms, which originate from quantum fluctuations, are engendered by either self-interactions of the chiral field or coupling to one or more different fields that play the role of a heat bath, provided one incorporates higher order terms in the computation of the effective equation of motion for $\phi(\vec{x}, t)$. In fact, it is well-known that one has to go up to two-loop corrections in order to pick up imaginary parts in the self-energy associated with viscosity and dissipation $[10,11,12,18]$. Selfinteractions of the $\phi$ field, as well as possible interactions with other fields in the medium, justify the inclusion of dissipation and noise terms such as done in Eq. (3) in the framework adopted here.

In our numerical simulations we solve Eq. (3) on a cubic space-like lattice. We use the leap frog method (see e.g. Refs. [15, 16]) for the time evolution and treat the Laplacian using a Fast Fourier Transform for the spatial dependence [19]. To minimize lattice artifacts, we use counterterms calculated in lattice perturbation in the classical theory (tadpole plus setting-sun diagrams) [20]:

$$
V_{C T}=\frac{1}{2}\left\{-3 \times 0.252731 \frac{T}{a}+\frac{6 T^{2}}{(4 \pi)^{2}}\left[\log \left(\frac{6}{\mu a}\right)+0.009\right]\right\} \phi^{2},
$$

where $T$ is the temperature, $a$ is the lattice spacing and $\mu$ is an arbitrary renormalization scale. We note that the addition of this counterterm is supposed to guarantee independence of the results on $a$ at equilibrium only. Initially we check the lattice independence of the results. We use units such that $m=1$ in the Lagrangian of Eq. (2), i.e. all dimensional quantities are expressed in units of $m$. In Fig. 1, we present the volume average of $\phi$ as a function of time, for (a) additive noise only, and (b) for additive plus multiplicative noises, for three different values of lattice spacings, $a=1 / 2,1 / 3$ and $1 / 4$, and a time step of $\Delta t=10^{-3}$. In these figures we fix the lattice extent to $L=16$, and use $T=1, \lambda=1$, and $\mu=1$. The initial configuration is taken to be a random distribution of the form $\phi(x, t)=\phi_{s p}+0.001 * \operatorname{rand}(\operatorname{seed})$, where $\phi_{s p}=1 / \sqrt{3}$ is spinodal point of the bare double-well potential, and $\mathrm{rand}(\mathrm{seed})$ is a uniform random number distribution in the interval $[-1,1]$. We average the results over several runs for different realizations of the noises. Clearly, as seen in the figure, the long-time (equilibrium) value of the $\langle\phi\rangle$ is fairly lattice independent. We have used other sets of parameters and obtained the same lattice independence of the equilibrium values of $\langle\phi\rangle$.

In Figure 2 we present preliminary results for different values of the multiplicative dissipation parameter $\eta_{2}$. As $\eta_{2}$ increases, the effect is of delaying the equilibration of the order parameter, as expected. This is, of course, in the same direction as with purely additive noise [8]. In Fig. 3 we investigate the effect of increasing the temperature. Of course, the higher the temperature, the larger the thermal fluctuations. Clearly seen in the figure is the restoration of the symmetry for temperatures between $T=3$ and $T=5$. Here we should note that $\eta_{1}$ and $\eta_{2}$ are functions of $T$ and so, increasing the 

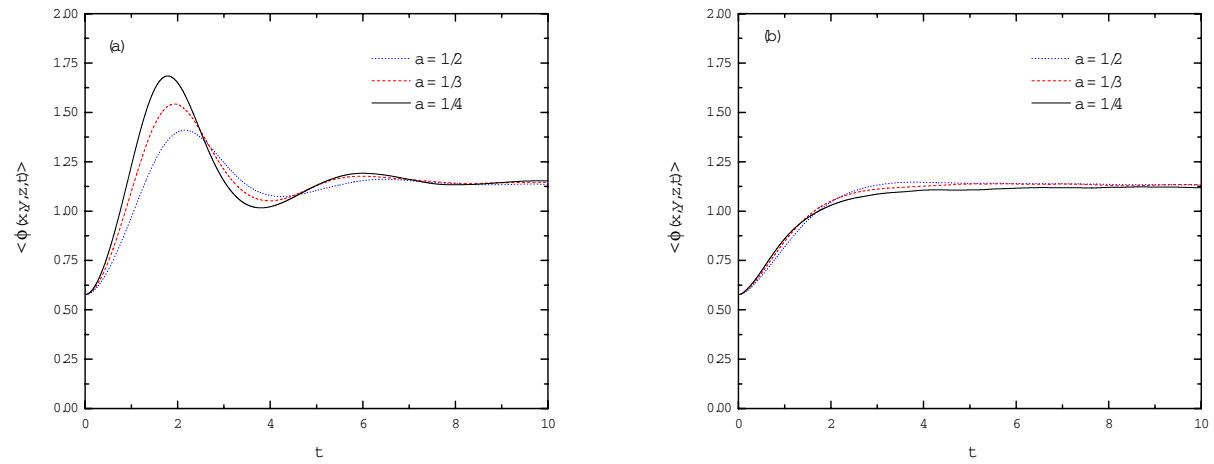

FIGURE 1. Volume average of $\phi(x, y, z, t)$ as a function of time for different lattice spacings, for temperature $T=1$ and counterterms scale $\mu=1$. (a) Additive noise only, $\eta_{1}=1, \eta_{2}=0$, (b) Additive plus multiplicative noises, $\eta_{1}=\eta_{2}=1$. Dimensional quantities are in units of $m$.

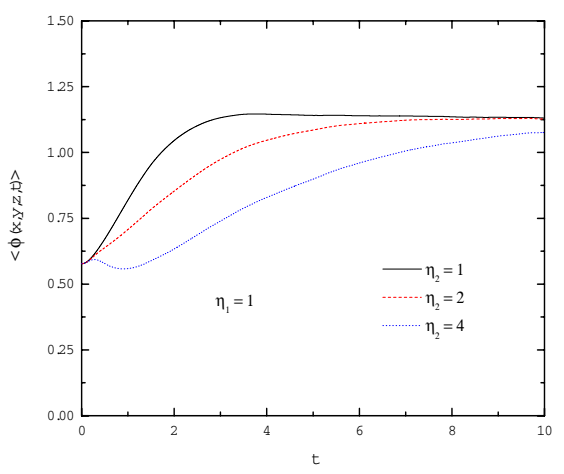

FIGURE 2. Volume average of $\phi(x, y, z, t)$ as a function of time for three different values of $\eta_{2}$, for $\eta_{1}=1$, temperature $T=1$, and counterterms scale $\mu=1$. Dimensional quantities are in units of $m$.

temperature and keeping these quantities fixed is not entirely consistent. Physically motivated parameterizations of $\eta_{1}(T)$ and $\eta_{2}(T)$ and a detailed study of the late-time behavior of the order parameter will be presented elsewhere [21].

\section{ACKNOWLEDGMENTS}

This work was partially supported by CAPES, CNPq, FAPERJ, FAPESP and FUJB/UFRJ. 

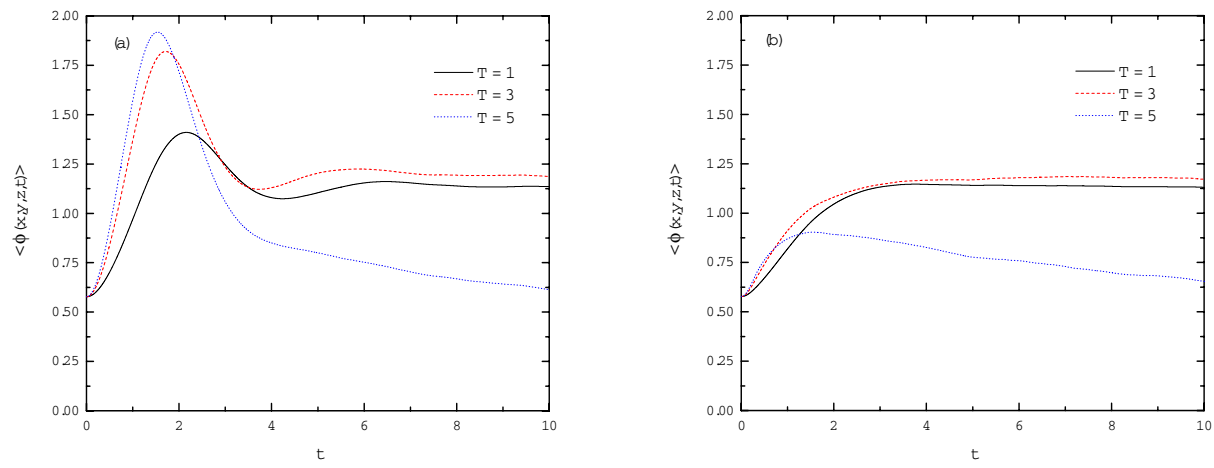

FIGURE 3. Volume average of $\phi(x, y, z, t)$ as a function of time for different lattice spacings, for three different temperatures, and counterterms scale $\mu=1$. (a) Additive noise only, $\eta_{1}=1, \eta_{2}=0$, (b) Additive plus multiplicative noises, $\eta_{1}=\eta_{2}=1$. Dimensional quantities are in units of $m$.

\section{REFERENCES}

1. J. D. Gunton, M. San Miguel and P. S. Sahni, in Phase Transitions and Critical Phenomena (Eds.: C. Domb and J. L. Lebowitz, Academic Press, London, 1983), vol. 8.

2. Proceedings of Quark Matter 2004, J. Phys. G 30 S633-S1425 (2004).

3. J. Rafelski and J. Letessier, Phys. Rev. Lett. 85, 4695 (2000).

4. A. Dumitru and R. D. Pisarski, Nucl. Phys. A 698, 444 (2002). O. Scavenius, A. Dumitru and A. D. Jackson, Phys. Rev. Lett. 87, 182302 (2001).

5. A. Dumitru and R. D. Pisarski, Phys. Lett. B 504, 282 (2001).

6. J. Randrup, Phys. Rev. Lett. 92, 122301 (2004); arXiv:nucl-th/0406031.

7. E. S. Fraga and R. Venugopalan, Physica A 345, 121 (2004).

8. E. S. Fraga and G. Krein, Phys. Lett. B 614, 181 (2005).

9. O. Scavenius, A. Dumitru, E. S. Fraga, J. T. Lenaghan and A. D. Jackson, Phys. Rev. D 63, 116003 (2001).

10. M. Gleiser and R. O. Ramos, Phys. Rev. D 50, 2441 (1994).

11. A. Berera, M. Gleiser and R. O. Ramos, Phys. Rev. D58, 123508 (1998).

12. D. H. Rischke, Phys. Rev. C 58, 2331 (1998).

13. R. O. Ramos and F. A. R. Navarro, Phys. Rev. D 62, 085016 (2000).

14. N. D. Antunes, P. Gandra and R. J. Rivers, Phys. Rev. D 71, 105006 (2005).

15. J. Borrill and M. Gleiser, Nucl. Phys. B 483, 416 (1997).

16. C. J. Gagne and M. Gleiser, Phys. Rev. E 61, 3483 (2000).

17. L. M. A. Bettencourt, K. Rajagopal and J. V. Steele, Nucl. Phys. A 693, 825 (2001).

18. A. Mocsy, Phys. Rev. D 66, 056010 (2002).

19. M. I. M. Copetti and C. M. Elliot, Mat. Sci. Tecn. 6, 273 (1990).

20. K. Farakos, K. Kajantie, K. Rummukainen and M. E. Shaposhnikov, Nucl. Phys. B 425, 67 (1994).

21. E. S. Fraga, G. Krein and R. O. Ramos, work in progress. 
Copyright of AIP Conference Proceedings is the property of American Institute of Physics and its content may not be copied or emailed to multiple sites or posted to a listserv without the copyright holder's express written permission. However, users may print, download, or email articles for individual use. 